\title{
Barriers and Facilitators of Using Quality Improvement To Foster Locally Initiated Innovation in Palliative Care Services in India
}

\author{
Karleen F. Giannitrapani, PhD, MPH, MA ${ }^{1,2}$ (D) Aanchal Satija, PhD, $M S^{3}$, \\ Archana Ganesh, MPH, MBA'3 , Raziel Gamboa, $M A^{1,2}$, Soraya Fereydooni, $B S^{1,2}$, \\ Taylor Hennings, $B S^{4}$, Shivani Chandrashekaran, $B S^{5}$, Jake Mickelsen, $M B A^{6}$, \\ Michelle DeNatale, $\mathrm{MSc}^{6}$, Odette Spruijt, MBChB, FRACP, FRAChPM7, \\ Sushma Bhatnagar, MD, MS ${ }^{3}$, and Karl A. Lorenz, MD, MSHS $S^{1,2}$
}

\begin{abstract}
${ }^{1}$ Center for Innovation to Implementation, VA Palo Alto Healthcare System, Menlo Park, CA, USA; ${ }^{2}$ Primary Care and Populaiton Health, Stanford University School of Medicine, Palo Alto, CA, USA; ${ }^{3}$ All India Institute of Medical Sciences (AllMS), New Delhi, India; ${ }^{4}$ University of California Berkeley School of Public Health, Berkeley, CA, USA; ${ }^{5}$ Duke University School of Medicine, Durham, NC, USA; ${ }^{6}$ Stanford Healthcare, Stanford, CA, USA; ${ }^{7}$ Peter MacCallum Cancer Center, Melbourne, VIC, Australia.
\end{abstract}

BACKGROUND: Quality improvement (QI) methods represent a vehicle for fostering locally initiated innovation cycles. We partnered with palliative care services from seven diverse practice settings in India to foster locally initiated improvement projects.

OBJECTIVE: To evaluate the implementation experiences of locally initiated palliative care improvement projects at seven diverse sites and understand the barriers and facilitators of using QI to improve palliative care in India.

PARTICIPANTS: We use a quota sampling approach to capture the perspectives of 44 local stakeholders in each of the following three categories (organizational leaders, clinic leaders, and clinical team members) through a semi-structured interview guide informed by the consolidated framework for implementation research (CFIR). We use standard qualitative methods to identify facilitators and barriers to using QI methods in seven diverse palliative care contexts.

RESULTS: Across all sites, respondents emphasized the following factors important in the success of quality improvement initiative: leveraging clinic level data, QI methods training, provider buy-in, engaged mentors, committed leadership, team support, interdepartmental coordination, collaborations with other providers, local champions, and having a structure for accountability. Barriers to using QI methods to improve palliative care services included lack of designated staff, high patient volume, resources, patient population geographic constraints,

Dr. Giannitrapani presented initial findings at the Academy Health Annual Research meeting in Washington DC, June 2, 2019, in a poster presentation entitled: Barriers and Facilitators of Using Quality Improvement Methods to Foster Locally Initiated Innovation in Palliative Care Services in India. Complete results were presented at the Indian Association of Palliative Care Annual Conference in Guwahati India on February 16, 2020.

Electronic supplementary material The online version of this article (https://doi.org/10.1007/s11606-020-06152-y) contains supplementary material, which is available to authorized users.

Received September 5, 2019

Accepted August 12, 2020

Published online September 8, 2020 general awareness and acceptance of palliative care, and culture.

CONCLUSIONS: Empowering local leaders and medical personnel to champion, design, and iterate using QI methods represents a promising powerful tool to spread palliative care services in developing countries.

KEY WORDS: quality improvement; implementation evaluation; palliative care; India; qualitative.

$\mathrm{J}$ Gen Intern Med 36(2):366-73

DOI: $10.1007 / \mathrm{s} 11606-020-06152-y$

(c) Society of General Internal Medicine 2020

\section{INTRODUCTION}

There is unmet need for palliative care (PC) services in the developing world. Globally an estimated 40 million people are currently in need of PC; this demand will continue to grow as a consequence of the rising burden of both population aging and non-communicable diseases. ${ }^{1-4}$

Home to one sixth of the world population, India is experiencing an epidemiological transition to increasing incidences of chronic diseases including cancer. ${ }^{5}$ Previously thought of as a relatively small concern, 2.25 million people are currently living with cancer in India, with the new rate predicted to double in the next 20 years. ${ }^{6-8}$ This is just a small part of an estimated 5.4 million people per year needing PC in India, $70 \%$ of whom will face distressing symptoms that remain unaddressed and undertreated. $5,6,9$

To address this growing need for PC services, there are over 1000 PC units in India; however, 90\% of these are located in a single state (Kerala) serving only $3 \%$ of the country's population. Less than $2 \%$ of those in need of PC in India receive it. ${ }^{6,}{ }^{10}$ Despite the existence of an increasing number of PC programs and PC trained providers, India still ranks at the bottom of the Quality of Death Index. ${ }^{9}$ The "Quality of Death" index measures the environment, 
availability, cost, and the quality of end-of-life care in 40 countries. 9

Low uptake of PC can be conceptualized as a healthcare quality issue. Over the last decade, various quality improvement (QI) efforts have been initiated in India to enhance the safety, effectiveness, efficiency, and equitability of care ${ }^{11,12}$ The rapidly increasing societal burden of chronic diseases makes now the moment to disseminate QI methods and tools thought out the India cancer and palliative care medical communities.

In 2017, we implemented PC-PAICE: Palliative CarePromoting Access \& International Cancer Experience. In PC-PAICE, physician mentors from the USA and Australia (Stanford University, Duke University, University of California, San Francisco (UCSF), Johns Hopkins University, University Technology Sydney (UTS), and Peter MacCallum Cancer Centre) partnered with Indian PC and cancer centers (All India Institute of Medical Sciences (AIIMS), MNJ Institute of Oncology and Regional Cancer Center, Tata Memorial Hospital, Thrissur Institute of Palliative Care, Homi Bhabha Cancer Hospital and Research Center, Trivandrum Institute of Palliative Sciences Cipla Palliative Care \& Training Center) to train a cohort of Indian leaders in QI LEAN-based methods. ${ }^{13}$ The PCPAICE curricula included baseline states, problem descriptions, outcomes measurement, intervention deployment, sustainability, and QI methods (e.g., run charts, fishbone, A3 process). ${ }^{14-16}$ All these intend to facilitate systematic problem-solving. Trainees were recruited from the Indian Association of Palliative Care members, and mentors were recruited from faculty of the US and Australian sites with PC oncology services. In addition to the online monthly curricula, teleconferences were held to create a shared learning environment. Moreover, all teams met in person in February 2018 for further didactics, team building, and problem-solving. ${ }^{14}$

PC-PAICE mentors worked with Indian clinician leads to develop locally initiated quality improvement efforts at seven sites throughout India. In this analysis, we evaluated the implementation experiences of providers and organizational leaders at the seven pilot sites involved in the PC-PAICE to understand the barriers and facilitators to using QI methods to improve PC delivery in India.

\section{METHODS}

\section{Setting}

Data for this analysis were collected as part of a greater effort to evaluate the implementation of the PC-PAICE initiative: a multi-site effort to foster locally initiated quality improvement methods in PC in India. With the goal of achieving maximum variation, the seven sites selected for participation in the PCPAICE project are geographically and structurally diverse. Practice sites included independent palliative care clinics, large academic institutions, and oncology hospitals. The table below summarizes the participating sites.

\begin{tabular}{|c|c|c|c|c|c|c|c|}
\hline $\begin{array}{l}\text { Name of } \\
\text { institution }\end{array}$ & $\begin{array}{l}\text { All India } \\
\text { Institute of } \\
\text { Medical } \\
\text { Sciences }\end{array}$ & $\begin{array}{l}\text { MNJ } \\
\text { Institute of } \\
\text { Oncology } \\
\text { and } \\
\text { Regional } \\
\text { Cancer } \\
\text { Center }\end{array}$ & $\begin{array}{l}\text { Tata } \\
\text { Memorial } \\
\text { Hospital }\end{array}$ & $\begin{array}{l}\text { Thrissur } \\
\text { Institute of } \\
\text { Palliative Care }\end{array}$ & $\begin{array}{l}\text { Homi Bhabha } \\
\text { Cancer Hospital } \\
\text { and Research } \\
\text { Center }\end{array}$ & $\begin{array}{l}\text { Trivandrum } \\
\text { Institute of } \\
\text { Palliative } \\
\text { Sciences }\end{array}$ & $\begin{array}{l}\text { Cipla Palliative } \\
\text { Care and } \\
\text { Training Center }\end{array}$ \\
\hline City and state & $\begin{array}{l}\text { New Delhi, } \\
\text { Delhi }\end{array}$ & $\begin{array}{l}\text { Hyderabad, } \\
\text { Telangana }\end{array}$ & $\begin{array}{l}\text { Mumbai, } \\
\text { Maharashtra }\end{array}$ & Thrissur, Kerala & $\begin{array}{l}\text { Vishakapatnam, } \\
\text { Andhra } \\
\text { Pradesh }\end{array}$ & $\begin{array}{l}\text { Trivandrum, } \\
\text { Kerala }\end{array}$ & $\begin{array}{l}\text { Pune, } \\
\text { Maharashtra }\end{array}$ \\
\hline Facility type & Government & Government & Government & $\begin{array}{l}\text { Charitable } \\
\text { Trust }\end{array}$ & Government & $\begin{array}{l}\text { Charitable } \\
\text { Trust }\end{array}$ & $\begin{array}{l}\text { Foundation } \\
\text { under CIPLA } \\
\text { Pharmaceutical } \\
\text { (CSR initiative) }\end{array}$ \\
\hline $\begin{array}{l}\text { Facility service } \\
\text { range }\end{array}$ & $\begin{array}{l}\text { Medical } \\
\text { College and } \\
\text { Public } \\
\text { University }\end{array}$ & $\begin{array}{l}\text { Oncology } \\
\text { Hospital }\end{array}$ & $\begin{array}{l}\text { Oncology } \\
\text { Hospital }\end{array}$ & Stand-alone PC & $\begin{array}{l}\text { Oncology } \\
\text { Hospital }\end{array}$ & $\begin{array}{l}\text { Stand-alone } \\
P C\end{array}$ & Stand-alone PC \\
\hline $\begin{array}{l}\text { Facility } \\
\text { characteristics } \\
\text { (facilities, beds) }\end{array}$ & $\begin{array}{l}\mathrm{OP}+\mathrm{IP}+ \\
\text { homecare }+ \\
\text { hospice } \\
1800 \text { beds }\end{array}$ & $\begin{array}{l}\mathrm{OP}+\mathrm{IP}+ \\
\text { homecare }+ \\
\text { hospice } \\
450 \text { beds }\end{array}$ & $\begin{array}{l}O P+I P+ \\
\text { hospice } \\
630 \text { beds }\end{array}$ & $\begin{array}{l}O P+I P+ \\
\text { homecare } \\
10 \text { beds }\end{array}$ & $\begin{array}{l}\text { OP + } \\
\text { homecare + } \\
\text { hospice }\end{array}$ & $\begin{array}{l}O P+I P+ \\
\text { homecare } \\
16 \text { beds }\end{array}$ & $\begin{array}{l}O P+I P+ \\
\text { homecare } \\
55 \text { beds }\end{array}$ \\
\hline QI project & $\begin{array}{l}\text { Improved } \\
\text { referral to } \\
\text { palliative } \\
\text { medicine for } \\
\text { advanced } \\
\text { oral cancer } \\
\text { patients }\end{array}$ & $\begin{array}{l}\text { Quality } \\
\text { improvement } \\
\text { in care } \\
\text { coordination } \\
\text { between } \\
\text { hospital and } \\
\text { hospice } \\
\text { based } \\
\text { palliative } \\
\text { care }\end{array}$ & $\begin{array}{l}\text { Institution of } \\
\text { a standard } \\
\text { process for } \\
\text { referral to } \\
\text { early } \\
\text { palliative } \\
\text { care for } \\
\text { patients with } \\
\text { advanced } \\
\text { lung cancer }\end{array}$ & $\begin{array}{l}\text { Documentation } \\
\text { of discussion } \\
\text { on prognostication }\end{array}$ & $\begin{array}{l}\text { Organizing } \\
\text { home } \\
\text { care services }\end{array}$ & $\begin{array}{l}\text { City } \\
\text { Homecare } \\
\text { Team, } \\
\text { Relax! } \\
\text { Palliative } \\
\text { protocols } \\
\text { are ready } \\
\text { for you }\end{array}$ & $\begin{array}{l}\text { Enabling early } \\
\text { palliative care } \\
\text { referral to } \\
\text { CIPLA } \\
\text { palliative care } \\
\text { center }\end{array}$ \\
\hline
\end{tabular}




\section{Ethics Approval}

This study was approved by both the Stanford University Institution review board (IRB - 42633) and the Institute Ethics Committee of the All India Institute of Medical Sciences (IEC572/03/11.2017, RP-41/2017).

\section{Approach Overview}

We conducted in-depth semi-structured interviews at the seven participating sites of the PC-PAICE initiative. The interview guide solicited feedback about participating in PCPAICE program and experiences of implementation. The implementation section of the guide was informed by the consolidated framework for implementation research (CFIR). ${ }^{17}$ The interview covered experience with PC-PAICE course content, experience participating in monthly group calls, experience at the in-person meeting, most important barriers you and your team faced in carrying out the QI project, challenges to making PC more accessible, most important opportunities for improving PC in your organization, and facilitators of QI and PC (see the interview guide).

\section{Participants}

We contacted a PC-PAICE champion from each site to request introductions to the stakeholders involved in the PC-PAICE QI project locally. Once the potential interviewees were identified, a member of our team contacted them via email and telephone to request participation. The site champion ultimately coordinated interview schedules, site visits, and space for the interview. In the case that geographical constraints prohibited in-person interviews, we allowed interviews to occur by phone. We employed a quota sampling approach to capture the perspectives of at least one stakeholder at each site in each of the following roles: organizational leaders, clinic leaders, and clinical team members (medical doctors, medical residents, nurses, and social workers). All participation was voluntary and completed with written informed consent. Most interviews (43/44) were audio-recorded except if a participant requested written notes only.

\section{Data Collection}

Our evaluation team is comprised of three PC physicians (KL, $\mathrm{OS}, \mathrm{SB})$, one qualitative methodologist $(\mathrm{KG})$, and multiple analysists. The qualitative methods expert trained two analysts based in India (AS, AG) in interviewing techniques prior to commencing data collection. All interviews were then attended by both analysts with one conducting the interview and the second taking detailed notes. All except one interview were digitally recorded and transcribed. We anonymized all interviews removing any personally identifiable information.

Key stakeholders from each of the seven sites were invited by email and phone to participate in interviews. Interviews were collected via site visits to five of the seven sites (sites A to E). Due to feasibility issues (e.g., regional flooding), interviews were scheduled via telephone or Zoom meeting for participants at sites $\mathrm{F}$ and $\mathrm{G}$. Two other interviews were conducted over the phone to accommodate stakeholder availability. A total of 44 interviews were conducted: organizational leaders $(n=8)$, clinical leaders $(n=12)$, and PC-PAICE participating members $(n=24)$.

\section{Codebook Development}

After each interview, the two analysts (AS, AG) created structured interview summaries that captured interview highlights as well as positive and negative experiences with PCPAICE. ${ }^{18}$ Interview summaries were reviewed by the entire evaluation team in a standing weekly meeting. The qualitative expert attended two of the seven site visits to provide the analysts with feedback on the interview and summary process. Interview summaries were consolidated into site summaries to identify emergent barriers and facilitators at each site. The summaries were used to inform the development of an initial code list.

\section{Data Analysis}

Three investigators (KG, AS, AG) then open coded one transcript and met to consolidate the open codes into a code list with systematic code definitions informed by the summaries. ${ }^{19}$ After coding three transcripts, consensus was reached around codes and definitions. This code list was then applied to all remaining transcripts by a single coder (one of RG, SF, AS, AG, TH, SC) and reviewed by a second coder. Bi-weekly team meetings were held over 4 months to manage coding discrepancies and come to consensus around code application. All analysis was conducted in Atlas.ti Version $8{ }^{20,}{ }^{21}$ The facilitators and barriers mentioned below are the ones that came up by at least one stakeholder at each of the seven sites.

\section{RESULTS}

A total of 44 interviews were conducted with organizational leaders $(n=8)$, clinic leaders $(n=12)$, and PC-PAICE participating clinical team members $(n=24)$.

\section{Facilitators}

Across all seven sites, respondents emphasized the importance of provider buy-in and comprehension of the transformational power of a systematic approach to using data to make improvements at the clinic level, assigned and engaged expert mentors, training on QI methods, committed leadership and team, interdisciplinary coordination, local champions, effective partnerships/collaborations with other providers, and having a structure for accountability as supportive of quality improvement initiative success. QI methods gave local clinicians a structure for accountability in that there were templates (e.g., for monitoring "how many patients are being registered newly and how many new patients the form has been completed in the 
prescribed manner"). Also notable, key stakeholders emphasized the attitude of the government towards palliative care has changed dramatically in recent years with improved funding, improved morphine access, and the presence of National Program for Palliative Care (NPPC) guidelines.

\section{Provider Buy-in and Comprehension of the Transformation-} al Power of a Systematic Approach to Using Data To Make Improvements at the Clinic Level. A leading facilitator for the adoption of QI across the diverse practice environments was providers realizing the power of what QI can do. This realization of the transformation power of a methodology goes beyond simple buy-in to a specific project. Providers and leaders felt empowered by "the realization that this methodology can be only not used for this goal [specific project], but it could be used for other goals... and that we could use this methodology and successfully reach the goals." Stakeholders described the transformation power of QI in diverse ways, the common thread being that it offers a strategy for moving towards solutions.

QI in itself kind of changes your thinking at the basic level like you don't just think of the problem...you think of the solution you work towards the solution. That kind of impacts our daily work... So, earlier I was just like is data that important but now I kind of understand why it is important. So, that way personally for my growth it was very helpful. Professionally, I think, having the group discussion as a team, and also involving hospice, involving hospital and then knowing that we were talking about making them the stakeholders. I don't think otherwise we would.

Assigned and Engaged Expert Mentors. Stakeholders across all roles were impacted by their assigned mentor. The qualities of a good mentor included being experienced in QI, efficient, involved in a continuous relationship over time, engaged, and proactive. The mentoring was perceived as effective when communication was reciprocal.

They were very experienced in QI project. They were very efficient mentors... So, we would all be connected together every week. And so there was a continuous exchange of what is happening, what are our doubts, problems and they would share, they would even engage... beyond this on emails. So, we had... informal, as well as very structured mentoring from them and lot of initiated from their side also.

\section{Training on a Straightforward and Replicable QI Method.} Different stakeholders preferred different QI approaches. However, most indicated that the thing that made the method helpful and able to be accomplished was when it was explained in a way they could understand.

now I know, it's [A3] a... sheet of paper with a template which tells a story and all the thanks to our mentor from Stanford... He helped understand that concepts of A3 in much much much better way... It's a very simple form of telling you a story.

Committed Leadership and Team. Stakeholders raised the importance of support at all levels starting from individual clinician buy-in to team and leadership support. No stakeholder said that taking on QI was easy. Many said it was challenging. A resounding sentiment was "What helps us is that we have, you know, a good dedicated, passionate team." Many highlighted the importance of having structured meetings on a predictable interval. In the face of poor provider buy-in or challenging team members, overwhelmingly team commitment and cohesion were the antidotes.

The reason it [QI project] was successful [despite poor initial buy-in] was because the interaction which we tried to ensure with the remaining team members... one core reason the project was successful was because the team was involved and they agreed to this change. QI team engagement was facilitated by team leader awareness and engagement. One of the roles of the team lead was to let the rest of the PC department know about the QI initiative to allow it to be incorporated in existing work culture and workflows.

We took the approval our institute to take part in this study... and because our team lead was aware that we were involved in this study, they gave us...the time and the resources to work on it and they got the team involved... because our team lead was making sure that our whole palliative care team is always aware that there is this project going on... it was easy for us to... get the work done.

Interdisciplinary Coordination. Multiple stakeholders highlighted the importance of interdisciplinary coordination. Examples demonstrated not only that interdisciplinary coordination supported QI but also that QI methods and processes furthered collaboration between frontline clinicians from nursing, medicine, and other disciplines.

Because of this project we did a series of academic discussions along with the goal of care... the nurses, the counsellors, the doctors... now our staff is much more tuned to this concept of identifying the goal of care for each patient... that kind of concept of care is more established now. 
Local Champions. Frontline clinicians from multiple practice contexts emphasized the importance of local champions in facilitating a successful QI project. Local champions broke down barriers between disciplines and brought diverse people together around shared goals.

I think my team lead is, you know, genius at this stuff and she has been able to... find people who share a common... interest in developing palliative care. One organizational leader highlighted how local champions were instrumental to initiative success through building effective partnerships and collaborations, not just with other providers but also in government and with non-governmental organizations (NGOs).

She's [Local Champion] been able to find key players in the government and in the NGO sector and she's been able to find...key funding sources... and mobilize all these sort of resources and to bring them together to work together for this vision [Improving palliative care].

\section{Barriers}

Barriers to using QI methods to improve PC services included "resources," "lack of designated staff," "high patient volume," patient population, "geographic constraints," general awareness and acceptance of PC, and cultural context (e.g., limited history of shared patientprovider decision-making, role of families, and stigma). Specific barriers to the QI methods also included "lack of project clarity" or specificity during the initial phase of the project and the dependency on other teams or team members for data and lack of electronic medical record infrastructure for sharing data. In the subject of resources, patient volume and staff time together influenced the ability of local clinics to take on QI effort. Organizational leaders and frontline clinicians alike highlighted how the project needed to be "an organizational priority from the leadership perspective"; otherwise, in the context of high patient volumes and the imperative to multi-task around the needs of multiple patients, frontline clinicians would feel QI was burdensome and not empowering.

Palliative Care Necessitates a Culture Shift in DecisionMaking. Barriers to using QI to improve PC include the patient and family expectations about their care and treatment. Many providers indicated that referring patients to PC represents a new approach for patients, and a culture shift needs to occur to engage them in the decision to choose PC options. Even when QI projects were successful in increasing referrals to PC, some faced the challenge of having the patient continuously return to the oncology department for additional treatment.

Patients... continue to ask for aggressive treatment, because they will have somebody in the family telling them that "no no you have to go for this" [palliative care] and they keep going back to parent department and they keep asking for some more... and then the parent department they are like ok you take one more radiation... and like this it keeps going on and on.Some PC providers expressed frustration with the fact that despite the understood appropriateness of PC, oncologists will still start treatments with curative intent.

... But I had a relative recently, whose wife was in the fourth stage... he knew that we are doing this thing, and then he came to me and I told him that this is going to be the end, and it will be much better if you choose palliative care... now he said fine but you know like when you are trying to clutch at straws... he went to the leading oncologist in the city... and then later on he told me that that gentleman told me exactly what you said that it won't work... but at the end of it, he said come on Monday and start the treatment... So that is the challenge. Further, a general "lack of awareness, lack of knowledge both in public and among the medical professionals" of PC hindered the success of QI projects. Awareness of the appropriateness of $\mathrm{PC}$ is critical so that it can hold a positive connotation and not mean "giving up fighting."

Generally among educated people I have seen that palliative care is almost like giving up for them... and the biggest challenge for them is to make them understand to stop unnecessary treatment which is not helping the patient... that carries a very negative connotation. Lack of awareness of the importance or value of PC could hinder the QI efforts if oncologists were unwilling to refer to a PC consult.

A brief session should be conducted for all the residents working in oncology about the palliative care and importance because... they [Oncologists] are busy in their stuff only...one good session with background and evidence of importance of palliative care, they will definitely consider sending the patients.

\section{DISCUSSION}

Empowering local leaders and medical personnel to champion, design, and iterate using QI methods represents a 
promising powerful tool to spread PC services in developing countries. This study provides insights into future initiatives as to what frontline clinicians found important and helpful. Frontline clinicians appreciated mentorship on QI methods when it was efficient and available over time. They further highlighted the powerful role of mentors in reducing QI methods down to a straightforward, replicable level. Especially in the context of vast patient loads and clinically complex patients, "simple" QI methods feel approachable. However, the majority of the facilitators identified focused on the local context and team. Stakeholders valued leadership support, leadership and team building, and the ability to collaborate and coordinate across disciplines. Established process for documentation, meeting, and collaboration were perceived as helpful. Similarly, lack of local resources, buy-in among leaders and team members, and clinician capacity were perceived as hindering the potential for successful QI adoption and spread.

Evaluating the spread of a palliative care related initiative gave us an opportunity to more deeply explore the cultural and informal caregiver influences on implementation efforts. In the American context, palliative care includes elements of supportive care such as therapeutic touch and acceptance of optimizing quality of life through providing pain and symptom management both during curative therapies and also at end of life. ${ }^{22}$ These constructs might need to be assimilated into the cultural context to be adopted. Providers in our study gave examples of how family members influence decisions towards more aggressive treatment. Family members may need to be socialized into palliative care to facilitate uptake.

From considering the examples given by providers in this study, we appreciate that future attention needs to be given to tailor PC approaches in general to the Indian cultural context. Lack of awareness, lack of knowledge, and lack of alignment with social norms will inhibit appreciation and hinder the success of the most wellmeaning QI projects. Facilitating uptake of PC may require more than communication and coordination as emphasized in CFIR and the Care Transitions Framework; it may also require tailoring and socializing to cultural norms and beliefs about pain, prognosis, and death. ${ }^{23,24}$

We built this collaboration between seven sites in India and expert international mentors to foster QI expertise in the PC community because we anticipated that many of the methods hold relevance across settings and country lines. After being exposed to QI, clinic leads from different sites mentioned feeling empowered to recognize the need for QI in other areas, e.g., systematically improving pain management through clinic population-level monitoring of pain scores. We believe expanding knowledge and capacity for QI can offer a promising strategy to address growing global PC needs from a population perspective.

This qualitative implementation evaluation demonstrated that PC-PAICE helped multiple sites establish processes to meet stated QI goals as well as brought new questions to the surface. We provided substantial expert mentoring on using a few specific QI projects in the context of PC clinics and we have anecdotal evidence from multiple sites that trained providers and section leads took these methods and applied them to address other PC concerns beyond their initial project as well as contexts outside of PC. This benefit of teaching QI methods should not be underestimated.

One of the principal challenges of international work is that so much of the time outsiders come from their home countries to the developing world with preconceived ideas about what should be implemented. ${ }^{25-27}$ Historically, many good lifesaving and quality-improving medical technologies have been developed and protocolized in one country and then exported to the developing world. Good PC, however, is necessarily contextual. $^{28-31}$ Culture, stigma, patient, and family expectations about experience of pain, illness, and death impact provider's perception of standards of care, conversations about goals of care, and treatment options offered at the end-of-life stage. ${ }^{33}$ Local and contextual resources also dictate the feasibility of care options. Teaching QI methods to the PC community in India and other developing contexts has the potential to be powerful because it truly facilitates context-based innovation. It can prevent imposing of external values and instead gives local physicians a toolbox they can draw on to innovate, design systemic changes, and transform clinical practices - removing all vestiges of the past from the spread of medical innovation.

\section{Limitations}

We acknowledge that this is just the first of many products to come from this portfolio of evaluation work; thus, it should be considered as a high-level look at themes holistically. We anticipate deep-dive analysis of each of the seven QI projects individually and deeper dives into the emergent themes. Specifically, the role of cultural context ${ }^{33}$ and government policies are underexplored in this work and each represents an area where we will publish an in-depth analyses in the future. In terms of other limitations, we acknowledge that there is some divergence from our initial protocol. We intended to make site visits to all seven sites to develop complete site summaries for triangulation purposes. However, due to severe flooding in southern India, it was impossible to travel to two of the sites during our data collection window. We were, however, able to do phone interviews with multiple respondents from those sites and feel the quality of the data collected is nearly equivalent to that collected during site visits. In fact, the quality of the recordings may have been even better with a dedicated phone line in comparison to some of the other places where interviews had to occur (e.g., noisy clinics without a private room). Instead of viewing this as a limitation, we conceptualize our protocol changes as a lesson for the necessity of adaptability when conducting high-quality qualitative evaluations in lower resourced or geographical disparate settings. 


\section{CONCLUSIONS}

This effort has salience far beyond PC in India. Through this project, we were able to build knowledge and capacity for QI, offering a promising strategy to address growing global PC needs from a population perspective. Early insights from this work indicate QI methods and mentoring may be a powerful and culturally sensitive approach for spreading locally initiated and tailored innovation or implementation in the developing world. Other initiatives to spread QI methods to improve quality of care are underway in diverse global settings. ${ }^{33-36}$ It is critical and time-sensitive to share learnings across medical disciplines and implementation contexts.

Acknowledgments: We would like to specifically acknowledge coauthors Michelle DeNatale, MSc, and Jake Mickelsen, MBA, of Stanford Healthcare for their contributions to developing the PCPAICE LEAN-based quality improvement. We would also like to thank all the clinicians and mentors who participated on the specific quality improvement projects at each of the seven sites.

Corresponding Author: Karleen F. Giannitrapani, $\mathrm{PhD}, \mathrm{MPH}, \mathrm{MA}$; Center for Innovation to Implementation, VA Palo Alto Healthcare System, Menlo Park, CA, USA (e-mail: Karleen@stanford.edu).

Funding This work was supported by a Conquer Cancer Grant from the ASCO foundation.

\section{Compliance with Ethical Standards:}

This study was approved by both the Stanford University Institution review board (IRB-42633) and the Institute Ethics Committee of the All India Institute of Medical Sciences (IEC-572/03/11.2017, RP-41/ 2017). All participation was voluntary and completed with written informed consent.

Conflict of Interest: Karleen Giannitrapani, Raziel Gamboa, Soraya Fereydooni, Taylor Hennings, Shivani Chandrashekaran, Odette Spruyt, Jake Mickelsen, Michelle DeNatale, and Karl Lorenz have no conflict of interest. Aanchal Satija, Archana Ganesh, and Sushma Bhatnagar report receiving support from Conquer Cancer, the ASCO foundation; this does not represent a conflict of interest. The findings and conclusions presented here are of the authors alone and do not represent Stanford University or the US Government.

\section{REFERENCES}

1. Gowshall M, Taylor-Robinson SD. The increasing prevalence of noncommunicable diseases in low-middle income countries: the view from Malawi. Int J Gen Med. 2018;11:255-264.

2. World Health Organization. Non communicable diseases. Available at: https://www.who.int/news-room/fact-sheets/detail/noncommunicable-diseases. June 1, 2018. Accessed August 13, 2019.

3. World Health Organization. Palliative care. Available at: https://www. who.int/news-room/fact-sheets/detail/palliative-care. February 19, 2018. Accessed August 13, 2019.

4. World Health Organization. WHO | 10 facts on noncommunicable diseases. WHO. Available at: https://www.who.int/features/factfiles/ noncommunicable_diseases/en/. March 2013. Accessed August 13, 2019.

5. Atreya S, Giri PA. Palliative care for cancer: a public health challenge in India. Peertechz Publications. 2017.

6. Jain V. Palliative care in India: trials, tribulations, and future prospects. Journal of Mahatma Gandhi Institute of Medical Sciences. 2018;23(2):55.
7. Ferlay J, Colombet M, Soerjomataram I, et al. Estimating the global cancer incidence and mortality in 2018: GLOBOCAN sources and methods. Int J Cancer. 2019;144(8): 1941-1953.

8. Broom A, Doron A. The rise of cancer in urban India: cultural understandings, structural inequalities and the emergence of the clinic. Health (London). 2012;16(3):250-266.

9. Kar SS, Subitha L, Iswarya S. Palliative care in India: situation assessment and future scope. Indian J Cancer. 2015;52(1):99-101.

10. Kumar S. Models of delivering palliative and end-of-life care in India. Current Opinion in Supportive and Palliative Care. 2013;7(2):216222.

11. Varkey $\mathbf{P}$, Kollengode A. A framework for healthcare quality improvement in India: the time is here and now!. J Postgrad Med 2011;57;237-41

12. Deorari. Delivery quality healthcare in India beginning of improvement journey. Indian Pediatr. 2018 Sep 15;55(9):735-737

13. Souza LBD. Trends and approaches in lean healthcare. Leadership in Health Services. 2009;22(2): 121-139.

14. Kiss-Lane T, Lorenz $\mathbf{K}$, DeNatale M, Mickelsen J. PC-PAICE Palliative Care-Promoting Access \& International Cancer Experience in India Collaborative (QI717). Journal of Pain and Symptom Management. 2019;57(2):465.

15. Shook J. Managing to Learn: Using the A3 Management Process to Solve Problems, Gain Agreement, Mentor and Lead. Cambridge, MA: Lean Enterprise Institute; 2008.

16. Cantiello J, Kitsantas P, Moncada S, Abdul S. The evolution of quality improvement in healthcare: patient-centered care and health information technology applications. Journal of Hospital Administration. 2016;5(2).

17. Damschroder LJ, Aron DC, Keith RE, Kirsh SR, Alexander JA, Lowery JC. Fostering implementation of health services research findings into practice: a consolidated framework for advancing implementation science. Implementation Science. 2009;4(1).

18. Creswell JW. Qualitative Inquiry and Research Design: Choosing among Five Approaches. SAGE Publications; 2013.

19. Glaser BG, Strauss AL. The Discovery of Grounded Theory: Strategies for Qualitative Research. Routledge; 2017.

20. Bernard HR. Research Methods in Anthropology: Qualitative and Quantitative Methods. AltaMira Press; 2002.

21. ATLAS.ti [computer program]. 8th Edition: Scientific Software Development; 2019.

22. Kelley AS, Morrison RS. Palliative care for the seriously ill. N Engl J Med. 2015;373(8):747-755.

23. Dy SM, Ashok M, Wines RC, Rojas Smith L. A framework to guide implementation research for care transitions interventions. The Journal for Healthcare Quality (JHQ). 2015;37(1):41.

24. Santesso N, Tugwell P. Knowledge translation in developing countries. J Contin Educ Health Prof. 2006;26(1):87-96.

25. Ebrahim S, Smith GD. Exporting failure? Coronary heart disease and stroke in developing countries. Int J Epidemiol. 2001;30(2):201-205.

26. Miranda JJ, Zaman MJ. Exporting "failure": why research from rich countries may not benefit the developing world. Rev Saude Publica. 2010;44(1): 185-189.

27. Ddungu H. Palliative care: what approaches are suitable in developing countries? British Journal of Haematology. 2011;154(6):728-735.

28. Stjernswärd J, Foley KM, Ferris FD. The Public Health Strategy for Palliative Care. Journal of Pain and Symptom Management. 2007;33(5):486-493.

29. Mathew-Geevarughese SE, Corzo O, Figuracion E. Cultural, Religious, and Spiritual Issues in Palliative Care. Prim Care. 2019;46(3):399-413.

30. Rahemi Z, Williams CL. Does ethnicity matter-cultural factors underlying older adults' end-of-life care preferences: a systematic review. Geriatr Nurs. July 2019.

31. Green A, Jerzmanowska N, Green M, Lobb EA. "Death is difficult in any language": a qualitative study of palliative care professionals' experiences when providing end-of-life care to patients from culturally and linguistically diverse backgrounds. Palliat Med. 2018;32(8): 1419-1427.

32. Raziel Gamboa, Sushma Bhatnagar, Aanchal Satija, et al. Myths that Inhibit the Spread of Palliative Care in India (S733). Journal of Pain and Symptom Management 2020;59(2):547.

33. Cavallin F, Maziku D, Mkolomi R, et al. Changes in maternal and neonatal care after a quality improvement intervention in a sub-Saharan setting. J Matern Fetal Neonatal Med. March 2019:1-7. 
34. Walley SC, Mussman GM, Lossius M, et al. Implementing parental tobacco dependence treatment within bronchiolitis QI collaboratives. Pediatrics. 2018;141(6):e20173072.

35. Kallam B, Pettitt-Schieber C, Owen M, Agyare Asante R, Darko E, Ramaswamy R. Implementation science in low-resource settings: using the interactive systems framework to improve hand hygiene in a tertiary hospital in Ghana. Int J Qual Health Care. 2018;30(9):724-730.
36. Manzi A, Mugunga JC, Nyirazinyoye L, et al. Cost-effectiveness of a mentorship and quality improvement intervention to enhance the quality of antenatal care at rural health centers in Rwanda. Int $J$ Qual Health Care. 2019;31(5):359-364.

Publisher's Note: Springer Nature remains neutral with regard to jurisdictional claims in published maps and institutional affiliations. 